\title{
The Optically Unbiased GRB Host (TOUGH) Survey
}

\author{
Páll Jakobsson $^{1}$, Jens Hjorth ${ }^{2}$, Daniele Malesani ${ }^{2}$, Johan P. U. \\ Fynbo $^{2}$, Thomas Krühler ${ }^{2}$, Bo Milvang-Jensen ${ }^{2}$ and Nial R. Tanvir ${ }^{3}$ \\ ${ }^{1}$ Centre for Astrophysics and Cosmology, Science Institute, University of Iceland, Dunhagi 5 , \\ 107 Reykjavík, Iceland \\ ${ }^{2}$ Dark Cosmology Centre, Niels Bohr Institute, University of Copenhagen, Juliane Maries Vej \\ 30, 2100 Copenhagen $\varnothing$, Denmark \\ ${ }^{3}$ Department of Physics and Astronomy, University of Leicester, University Road, Leicester, \\ LE1 7RH, UK
}

\begin{abstract}
We present the results from our Swift/VLT legacy survey, a VLT Large Programme aimed at characterizing the host galaxies of a homogeneously selected sample of Swift gammaray bursts (GRBs). The immediate goals are to determine the host luminosity function, study the effects of reddening, determine the fraction of Ly $\alpha$ emitters in the hosts, and obtain redshifts for targets without a reported one. We have carefully selected a sample, obeying strict and welldefined criteria: 69 targets in total. Among the results is a large optical detection rate, the lack of extremely red objects (only one possible case in the sample), and 15 new GRB redshifts with the mean redshift of the host sample assessed to be $\langle z\rangle \gtrsim 2.2$.
\end{abstract}

Keywords. dust, extinction, galaxies: fundamental parameters, gamma rays: bursts

\section{Introduction}

Determining the statistical properties of gamma-ray bursts (GRBs) has long been compromised by inhomogeneous selection and a bias against optically dark bursts. With Swift (Gehrels et al. 2004) it has become possible to construct much more uniform samples, and to target the host galaxies even of optically faint bursts via X-Ray Telescope (XRT) localizations, for which redshifts could not be determined from the afterglows.

We have been securing GRB host galaxy information for a homogeneous sample of 69 Swift GRBs with a large programme at the Very Large Telescope (VLT). We aim at selecting a representative, unbiased sample of GRB host galaxies. To optimize the survey, we focus on the systems with the best observability, which also have the best available information. We thus select Swift bursts with the following properties:

- A long-duration burst detected automatically by the Burst Alert Telescope.

- The Galactic extinction in the direction to the burst has to be $A_{V} \leqslant 0.5$ mag.

- The Sun-to-field distance, at the time of the GRB, has to be $\theta_{\text {Sun }} \geqslant 55^{\circ}$.

- Good observability from the VLT $\left(-70^{\circ}<\delta<+27^{\circ}\right)$.

- Prompt XRT localization distributed within 12 hours.

- Happened between March 2005 and August 2007.

- A small XRT error circle (radius $\leqslant 2^{\prime \prime}$ ).

Imposing these restrictions does not bias the sample towards optically bright afterglows; instead each GRB in the sample has favourable observing conditions, i.e. useful follow-up observations are likely to be secured. The bursts fulfilling all the criteria, 69 targets in total, make up The Optically Unbiased GRB Host (TOUGH) sample. More than $75 \%$ of the sample have an optical/near-IR afterglow (OA) (52/69) and 55\% already 

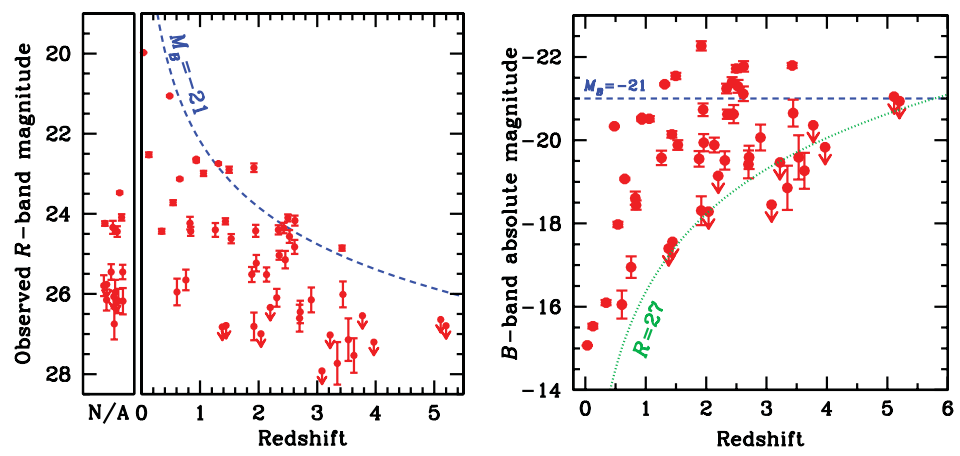

Figure 1. Left: The $R$-band host magnitude as a function of redshift for all the bursts in the TOUGH sample. Upper limits are shown with arrows. Hosts without a reported redshift are plotted on the left side of the diagram. The dashed curve shows a galaxy with an absolute $B$-band magnitude of -21 assuming $F_{\nu} \propto \nu^{-0.5}$. Right: The absolute $B$-band magnitude $\left(F_{\nu} \propto \nu^{-0.5}\right)$ as a function of redshift for all the hosts in the left panel with a reported redshift. The dotted curve shows a galaxy with an observed magnitude of $R=27$.

have a reported reliable redshift $(38 / 69)$ in the range $0.033<z<6.3$. This should be compared to the full Swift sample that only has around $30 \%$ redshift completion.

\section{Host magnitudes and colours}

The first step in the TOUGH program is to obtain sufficiently deep images of the targets in the $R$ - (typically restframe UV) and $K$-band (typically restframe visual). The characteristic magnitude limits of the survey are $R \sim 27$ and $K \sim 21.5$ (Vega photometric system). Around $80 \%$ (54/69) of the hosts are detected in the $R$ band (Fig. 1), being mostly subluminous (between $1 \%$ and roughly $100 \%$ of $L^{*}$ ) as previous findings have indicated (e.g. Le Floc'h et al. 2003; Fruchter et al. 2006). HST observations have recently been carried out to search for some of the missing hosts in the sample.

Could a large fraction of faint hosts go undetected in our survey? The probability for a galaxy to be detected within an afterglow error circle by chance depends on the magnitude of the galaxy. The number of galaxies per $\operatorname{arcmin}^{2}$ has been well determined to deep limits in the various Hubble deep fields. To limits of $R=24,26$ and 28 there are about 2, 6 and 13 galaxies $\operatorname{arcmin}^{-2}$ (e.g. Fynbo et al. 2000). Hence, the probability to find an $R=24$ galaxy by chance in an error circle with a $0 \prime .5$ radius is about $0.4 \%$. For a $R=28$ galaxy the probability is about $8 \%$. If the error circle is defined only by the X-ray afterglow with a $2^{\prime \prime}$ radius we expect a random $R=24$ and $R=28$ galaxy in $6 \%$ and $72 \%$ of the error boxes. There are 17 bursts with X-ray positions only in the TOUGH sample ( $75 \%$ have an OA). Of these the faintest host has $R \sim 26$. Hence, chance projection should hence not be a serious concern and we are confident that the vast majority of the host candidates are real.

Only $35 \%$ of the hosts are detected in the $K$ band. The corresponding $R-K$ color is shown in Fig. 2 with the hosts predominantly lying in the range $2<R-K<5$. There is only a single possible extremely red object (ERO) with $R-K=5.6$; this GRB had no reported OA to a limit of around $R>24$ at 1 hour after the burst (Fynbo et al. 2009). Although this might indicate the presence of dust, a chance association cannot be excluded between the GRB and this galaxy, given that the XRT error circle is among the largest in the sample. Figure 2 also confirms earlier findings (e.g. Le Floc'h et al. 2003) that GRB hosts mostly have blue colors (even considering bursts with no reported OA). 


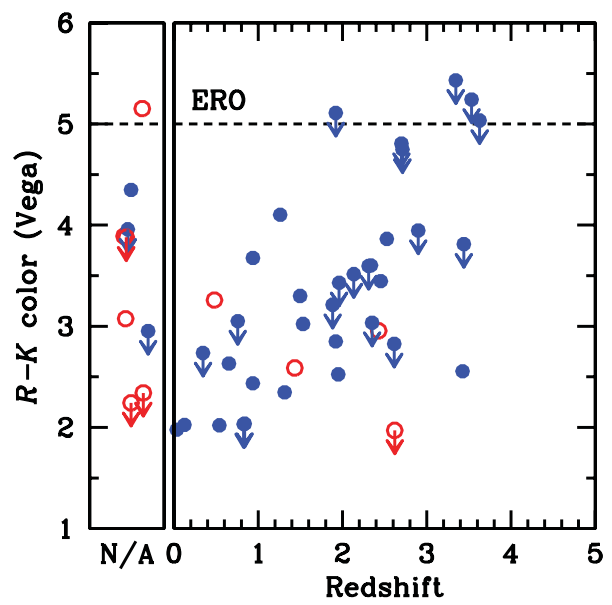

Figure 2. The $R-K$ color of the TOUGH hosts as a function of redshift. The left side represents objects without, as yet, a measured redshift. The filled (open) circles represent host galaxies of GRBs with (without) a detected OA. Only one ERO $(R-K>5)$ is visible in the sample.

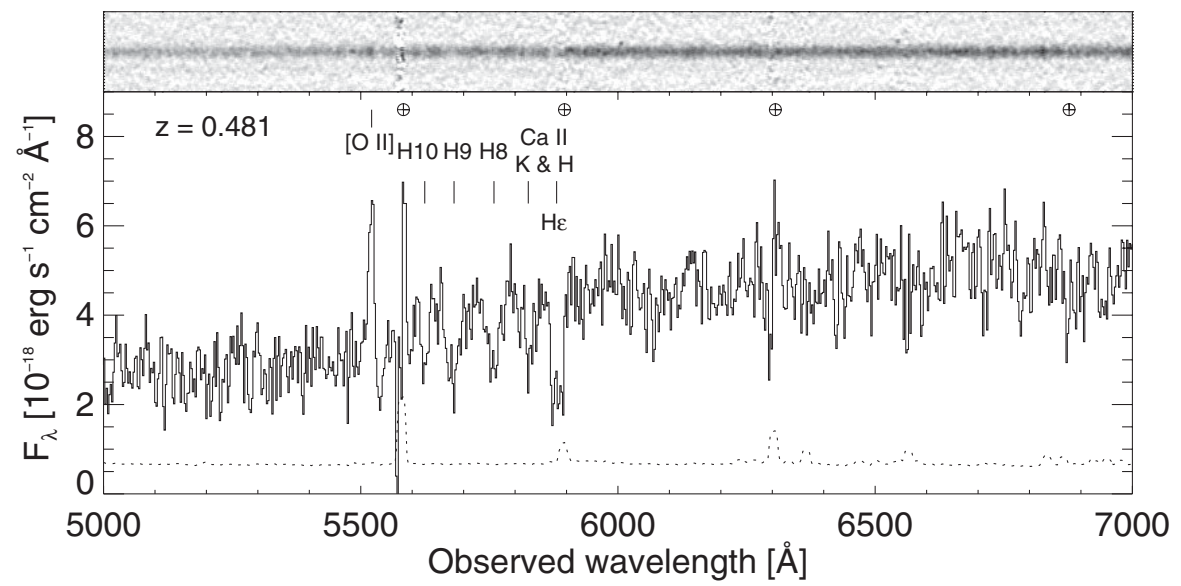

Figure 3. One- and two-dimensional spectra of a host galaxy in the TOUGH sample. Line features are marked with vertical lines, whereas telluric features and skyline residuals are marked with $\oplus$. The error spectrum is plotted as a dotted line.

\section{Spectroscopic observations}

We attempted spectroscopic observations of all hosts with $R \lesssim 25.5$ that did not have a reported reliable redshift. We have determined the redshift of 15 GRB host galaxies (an example is shown in Fig. 3) whose average redshift is $\langle z\rangle=1.8$, significantly lower than the overall Swift GRB mean redshift. The low value is most likely the result of targeting the brightest galaxies in the sample. Furthermore, we have estimated redshift limits for an additional five hosts and inferred that three burst redshifts reported in the literature are erroneous (GRBs 060306, 060814 and 060908). Figure 4 shows how the redshift distribution has changed due to our measurements. For more details about the spectroscopic results we refer to Jakobsson et al. (2012), Krühler et al. (2012) and Milvang-Jensen et al. (2012). 

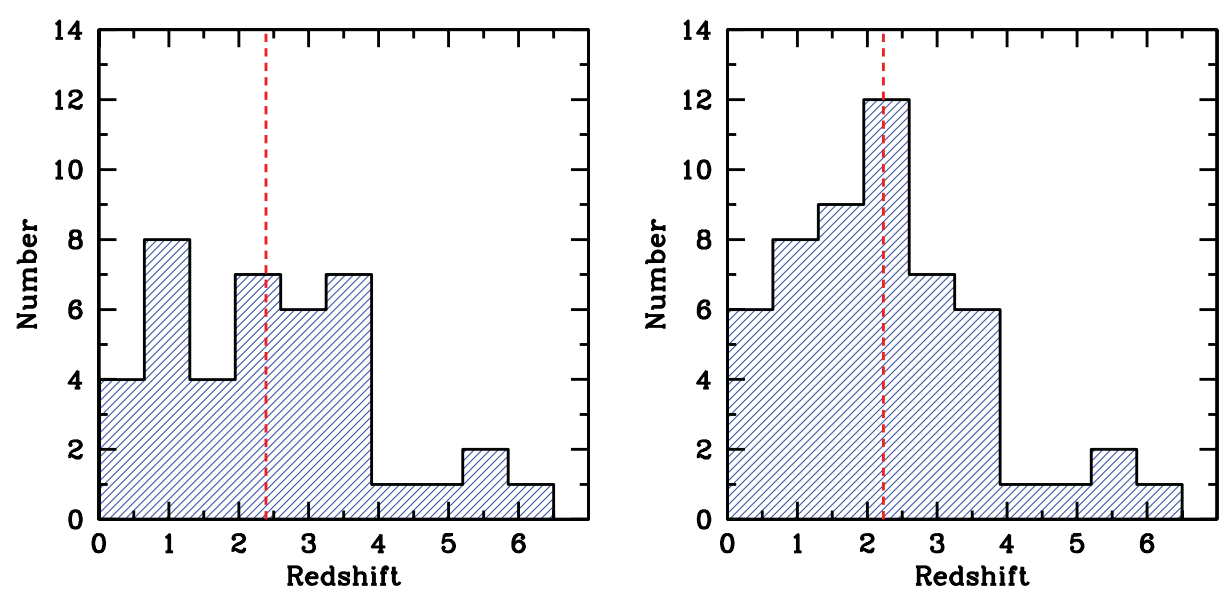

Figure 4. The redshift distribution of the TOUGH sample. In both panels the dashed vertical line is the mean redshift. Left: Before we started our observations, 38 redshifts were considered secure with $\langle z\rangle=2.39$. Right: Our TOUGH spectroscopic observations added 15 new redshifts and demonstrated that three redshift reported in the literature were erroneous. Here $\langle z\rangle=2.23$.

\section{Implications and prospects}

We have now reached a point in GRB research where a single burst rarely elucidates and illuminates our general understanding of the field. It is important to focus on well-defined samples and population studies, where systematics and biases can be minimized. Swift has made it possible to build such a sample and thanks to new available instrumentation, such as the VLT/X-shooter (Vernet et al. 2011), we can continue to follow this track into the future.

\section{References}

Gehrels, N., et al. 2004, ApJ, 611, 1005

Le Floc'h, E., et al. 2003, A\&BA, 400, 499

Fruchter, A. S., et al. 2006, Nature, 441, 463

Fynbo, J. P. U., Freudling, W., \& Møller, P. 2000, A\& $A, 355,37$

J. P. U. Fynbo, et al. 2009, ApJS, 185, 526

Jakobsson, P., et al. 2012, ApJ, in press

Krühler, T., et al. 2012, ApJ, submitted

Milvang-Jensen, B., et al. 2012, ApJ, submitted

Vernet, J., et al. 2011, A\&A, 536, 105

\section{Discussion}

AstraAtmadja: Based on the redshift distribution, what can we tell about the starformation history of the Universe?

JAKOBSSON: It is possible that star formation at high redshifts has been significantly underestimated. Even at $z \sim 2$ it appears that the galaxy luminosity function has a substantially steeper faint-end slope than locally. Alternatively, it could be that GRB production is substantially enhanced in the conditions of early star formation, beyond the metallicity-dependent rate correction already applied. In the long run, large complete samples of GRB redshifts should shed light on whether the GRB rate is proportional to SFR or whether other effects play an important role. 